\title{
EDITORIAL | PUBLICAÇÃO RESPONSÁVEL DA PESQUISA: PADRÕES INTERNACIONAIS PARA AUTORES
}

Durante a $2^{\text {a }}$ Conferência Mundial sobre Integridade da Pesquisa de Singapura, em 22 a 24 de julho de 2010, o Committee on Publication Ethics (COPE ${ }^{1}$ ) ajudou a desenvolver duas declarações que estabelecem padrões internacionais para publicação de pesquisa responsável para autores e editores.

Os documentos foram publicados como parte dos procedimentos da Conferência sob uma licença Creative Commons. O COPE encoraja editores a republicar as declarações em suas próprias revistas e a fazer referência ou a incorporá-las nas suas políticas e instruções editoriais para os autores.

Neste v. 19, n. 1, de 2018 da EJJL publicaremos a tradução e a versão original em inglês dos padrões recomendados para AUTORES. No v. 19, n. 2, publicaremos a tradução e a versão original dos padrões recomendados para EDITORES.

A EJJL2 vai incorporar essas orientações na sua política editorial.

Boa leitura.

Carlos Luiz Strapazzon

Editor-Chefe

Abril/2018

\footnotetext{
1 O COPE foi criado em 1997 por um pequeno grupo de editores de revistas no Reino Unido, mas atualmente tem mais de 12.000 membros em todo o mundo, de todas as áreas do conhecimento científico. A adesão está aberta a editores de revistas acadêmicas e outros interessados em ética de publicação. O COPE fornece conselhos a casas editoriais e a editores sobre todos os aspectos da ética da publicação e, em particular, sobre como lidar com casos de conduta indevida na pesquisa e em publicações. Não investiga casos individuais. O COPE financia pesquisas, organiza seminários anuais em nível mundial e também criou uma ferramenta de auditoria para que os membros avaliem suas políticas e práticas contra os princípios de publicação do COPE, descritos nas Práticas básicas. Mais informações, ver: <https://publicationethics.org/about >.

2 Espaço Jurídico Journal of Law (<https://editora.unoesc.edu.br/index.php/espacojuridico/index $>$ ).
} 


\section{Publicação responsável da pesquisa: padrões internacionais para autores ${ }^{3}$}

Declaração de posição desenvolvida na $2^{\text {a }}$ Conferência Mundial sobre Integridade da Pesquisa, Singapura, de 22 a 24 de julho de 2010

Elizabeth Wager \& Sabine Kleinert ${ }^{4}$

\section{Para citar a versão original em inglês:}

Wager E \& Kleinert S (2011) Responsible research publication: international standards for authors. A position statement developed at the 2nd World Conference on Research Integrity, Singapore, July 22-24, 2010. Chapter 50 in: Mayer T \& Steneck N (eds) Promoting Research Integrity in a Global Environment. Imperial College Press / World Scientific Publishing, Singapore (pp 309-16). (ISBN 978-981-4340-97-7)

\section{Resumo}

- A pesquisa deve ser conduzida de forma ética e responsável e deve observar todas as regras relevantes.

- Pesquisadores devem apresentar seus resultados de forma clara, honesta e sem fabricação, falsificação ou manipulação inadequada de dados.

- Pesquisadores devem se esforçar para descrever seus métodos de forma clara e inequívoca para que suas descobertas possam ser confirmadas por outros pesquisadores.

- Pesquisadores devem respeitar os requisitos específicos de uma publicação, ou seja, que o trabalho seja original, não plagiado e ainda não publicado noutro lugar.

- Coautores devem assumir a responsabilidade coletiva pelo trabalho submetido a publicação e por trabalhos publicados.

- A autoria de publicações deve refletir precisamente todas as contribuições individuais para o resultado da pesquisa e para o conteúdo de relatórios.

- Devem ser identificados os financiadores da pesquisa e informados eventuais conflitos de interesse.

\footnotetext{
Disponível em: < https://publicationethics.org/node/11184>. A permissão para republicação não é necessária. A autorização das autoras para publicar a versão em língua portuguesa foi concedida em 18 de abril de 2018.

Esta tradução foi finalizada em 11 de abril de 2018 por Carlos Luiz Strapazzon, Editor-Chefe da EJJL, Prof. de Direito Constitucional do PPGD-UNOESC e na Universidade Positivo, PR, Brasil. E-mail: strapazzon.carlos.luiz@gmail.com; Academia: https://cstrapazzon.academia.edu; Bepress: works.bepress.com/carlos_strapazzon

4 Contatos: liz@sideview.demon.co.uk; sabine.kleinert@lancet.com
} 


\section{Introdução}

A publicação é a fase final da pesquisa e, portanto, é responsabilidade de todos os pesquisadores. As publicações acadêmicas devem fornecer um registro detalhado e permanente da pesquisa. Como as publicações formam a base para novas pesquisas e para a aplicação das descobertas, podem afetar não somente a comunidade de pesquisa, mas também, indiretamente, a sociedade em geral. Os pesquisadores têm, portanto, a responsabilidade de garantir que suas publicações sejam honestas, claras, precisas, completas e equilibradas, e devem evitar relatórios enganosos, seletivos ou ambíguos. Os editores de revistas também têm responsabilidades para garantir a integridade da literatura de pesquisa, e estas são estabelecidas em diretrizes complementares.

Este documento tem como objetivo estabelecer padrões internacionais para autores de publicações de pesquisas acadêmicas e também descrever práticas responsáveis para a elaboração de relatórios de pesquisa. Espera-se que esses padrões sejam endossados por instituições de pesquisa, financiadores e sociedades profissionais; que seja promovido por casas editoriais e por editores; e que possa ser útil na capacitação para a integridade na pesquisa.

\section{Publicação responsável de pesquisa}

\section{Solidez e confiabilidade}

1.1 A pesquisa relatada deve ter sido realizada de forma ética e responsável e com observação de toda a legislação relevante.

1.2 A pesquisa relatada deve ser consistente e deve ter sido cuidadosamente executada.

1.3 Pesquisadores devem usar métodos apropriados de análise e de apresentação de dados (e, se necessário, buscar e seguir recomendações de especialistas).

1.4 Autores devem assumir a responsabilidade coletiva pelo trabalho e pelo conteúdo de suas publicações. Pesquisadores devem verificar cuidadosamente suas publicações em todas as etapas para garantir que os métodos e resultados sejam relatados com precisão. Autores devem verificar com cuidado os cálculos, apresentações de dados e versões preliminares.

\section{Honestidade}

2.1 Pesquisadores devem apresentar seus resultados com honestidade e sem fabricação, falsificação ou manipulação inadequada de dados. Imagens de pesquisas não devem ser modificadas de forma enganosa. 
2.2 Pesquisadores devem se esforçar para descrever seus métodos e apresentar seus resultados de forma clara e inequívoca. Pesquisadores também devem seguir as diretrizes aplicáveis de elaboração de relatórios. As publicações devem fornecer detalhes suficientes para permitir que as experiências relatadas sejam repetidas por outros pesquisadores.

2.3 Os relatórios de pesquisa devem estar completos. Não devem omitir achados ou resultados inconvenientes, inconsistentes ou inexplicáveis que não confirmam a hipótese ou a interpretação dos autores ou de financiadores.

2.4 Pesquisadores e financiadores não devem vetar a publicação de descobertas que não favorecem seu produto ou posição. Pesquisadores não devem fazer acordos que permitam ao financiador da pesquisa vetar ou controlar a publicação de resultados (a menos que existam circunstâncias excepcionais, como ocorre com a pesquisa classificada por governos nos casos relacionados com segurança).

2.5 Autores devem alertar prontamente os editores de uma publicação se descobrirem um erro em qualquer trabalho submetido, aceito ou já publicado. Autores devem cooperar com editores na realização de correções ou retratações, quando necessário.

2.6 Autores devem apresentar o trabalho de outros pesquisadores com precisão em citações e referências.

2.7 Autores não devem copiar referências de outras publicações se não leram o trabalho citado.

\section{Equilíbrio}

3.1 Novas descobertas devem ser apresentadas no contexto de pesquisas anteriores. $\bigcirc$ trabalho de outros pesquisadores deve ser representado de forma justa. Avaliações acadêmicas e sínteses de pesquisa já existente devem ser completas, equilibradas e devem incluir descobertas mesmo se contrariam a hipótese ou interpretação que está sendo proposta. Editorais ou textos de opinião que apresentam um único ponto de vista ou argumento precisam ser claramente diferenciados dos trabalhos acadêmicos.

3.2 Todas as limitações da pesquisa e dos resultados devem ser explicadas nas publicações. 


\section{Originalidade}

4.1 Autores devem aderir aos requisitos de submissão sobre a originalidade do trabalho e assumir que apresentam um trabalho original e que não foi publicado em nenhum outro idioma. $\bigcirc$ trabalho não deve ser enviado simultaneamente a mais de uma publicação, a menos que os editores concordem com a copublicação. Se os artigos forem copublicados, esse fato deve ser esclarecido para os leitores.

4.2 Leis e convenções de direitos autorais devem ser observadas. O material sujeito a direitos autorais (como tabelas, figuras ou citações extensas) deve ser reproduzido apenas se tiver permissão e reconhecimento apropriado.

4.3 Relevantes trabalhos e publicações anteriores, de outros pesquisadores ou dos próprios autores, devem ser devidamente reconhecidos e referenciados. A literatura primária deve ser citada sempre que possível.

4.4 Dados, textos, figuras ou ideias criadas por outros pesquisadores devem ser devidamente reconhecidos e não devem ser apresentados como se tivessem sido elaborados pelos próprios autores. Formulações originais obtidas diretamente de publicações de outros pesquisadores devem aparecer entre aspas e com as citações apropriadas.

4.5 Autores devem informar os editores se os resultados foram publicados anteriormente ou se vários relatórios ou análises múltiplas de um único conjunto de dados estão em consideração para publicação em algum outro veículo de publicação. Autores devem fornecer cópias de publicações relacionadas ao trabalho submetido ou de outro trabalho já submetido a outras revistas.

4.6 Várias publicações decorrentes de um único projeto de pesquisa devem ser claramente identificadas, e a publicação primária deve ser devidamente referenciada nas demais. Traduções e adaptações para diferentes públicos devem ser claramente identificadas como tal, devem reconhecer a fonte original e respeitar as convenções relevantes de direitos autorais e de requisitos de permissão. Em caso de dúvida, os autores devem solicitar permissão ao editor original antes de republicar qualquer trabalho.

\section{Transparência}

5.1 Devem ser divulgadas todas as fontes de financiamento da pesquisa, inclusive o apoio financeiro direto e indireto, o fornecimento de equipamentos ou materiais, e outros apoios (como no caso do serviço especializado em estatística ou de assistência de redação). 
5.2 Autores devem divulgar o papel do(s) financiador(es) da pesquisa ou de patrocinadores (se houver) na delimitação do projeto, na execução, na análise, na interpretação e na elaboração de relatórios.

5.3 Autores devem divulgar interesses financeiros e não financeiros relevantes, bem como relacionamentos que possam ser considerados susceptíveis de afetar a interpretação de suas descobertas ou que editores, revisores ou leitores podem razoavelmente desejar saber. Isso inclui qualquer relação com a revista, por exemplo, se os editores publicarem suas próprias pesquisas em seu próprio periódico. Além disso, autores devem observar todos os requisitos estabelecidos pelos periódicos (ou pelas instituições) para divulgar interesses concorrentes.

\section{Autoria e reconhecimento apropriado de autoria}

6.1 A literatura de pesquisa registra não apenas o que foi descoberto, mas também quem fez a descoberta. Portanto, a autoria de publicações de pesquisa deve refletir com precisão as contribuições de cada indivíduo para os resultados de um trabalho e para o relatório final da pesquisa.

6.2 Nos casos em que os principais colaboradores serão listados como autores e os que fizerem contribuições menos substanciais ou puramente técnicas para a pesquisa, ou para a publicação, serão listados numa seção de reconhecimento, os critérios de autoria e de reconhecimento devem ser combinados antes do início do projeto de pesquisa. O ideal é que os critérios de autoria para uma área específica do conhecimento sejam acordados, publicados e aplicados de forma consistente por instituições de pesquisa, sociedades profissionais e acadêmicas e por financiadores. Na medida em que editores de periódicos devem publicar e promover critérios de autoria aceitos como apropriados para suas áreas, não se pode esperar que se pronunciem sobre questões de autoria. A responsabilidade pela atribuição correta da autoria é dos próprios autores que, por sua vez, devem trabalhar sob a orientação de sua instituição. As instituições de pesquisa devem promover e defender padrões justos e aceitáveis de autoria e de reconhecimento. Quando necessário, as instituições devem julgar as disputas sobre autoria e devem assegurar que o devido processo seja observado.

6.3 Pesquisadores devem garantir que somente os que atendem aos critérios de autoria (ou seja, que tenham contribuído substancialmente para o trabalho) sejam contemplados como autores e também que pesquisadores que merecem ser reconhecidos como autores não sejam omitidos. Instituições e editores de revistas devem encorajar práticas que impedem a autoria honorária (guest authorship), a autoria graciosa (gift authorship) e a autoria fantasma (ghost authorship). 
Notas:

- Autores honorários são aqueles que não cumprem critérios de autoria, mas estão listados por sua antiguidade, reputação ou suposta influência.

- Autores graciosos são aqueles que não cumprem critérios de autoria, mas estão listados por motivos de favorecimento pessoal ou em troca do pagamento.

- Autores fantasmas são aqueles que cumprem critérios de autoria, mas não estão listados.

6.4 Todos os autores devem concordar em estar listados e devem aprovar as versões enviadas e aceitas da publicação. Qualquer alteração na lista de autores deve ser aprovada por todos os autores. $\bigcirc$ autor responsável pelas correspondências deve atuar como ponto de contato entre editores e demais autores e deve manter os demais devidamente informados e envolvê-los quando for preciso tomar decisões importantes sobre a publicação (por exemplo, as respostas aos comentários de revisores).

6.5 Autores não devem usar o espaço dos agradecimentos para, de forma enganosa, associar uma certa contribuição ou endosso de resultados a indivíduos que, de fato, não se envolveram com o trabalho ou não endossaram os resultados.

\section{Accountability e responsabilidade}

7.1 Todos os autores devem ler e estar familiarizados com o texto do relatório final e devem garantir que as publicações observam as diretrizes estabelecidas neste Guia. Na maioria dos casos, espera-se que autores assumam a responsabilidade pela integridade da pesquisa e dos relatórios. No entanto, se autores assumem a responsabilidade apenas por certos aspectos da pesquisa e de relatórios, isso deve ser especificado na publicação.

7.2 Autores devem colaborar com os editores ou com a casa editorial para prontamente corrigir o trabalho quando, após a publicação, forem identificados erros ou omissões.

7.3 Autores devem observar convenções, requisitos e regulamentos relevantes para disponibilizar materiais, reagentes, softwares ou bases de dados a outros pesquisadores. Pesquisadores, instituições e financiadores devem ter políticas claras para lidar com esse tipo de pedidos. É recomendável, também, seguir os padrões de periódicos científicos relevantes. Embora o reconhecimento adequado seja esperado, pesquisadores não devem reivindicar coautoria como uma condição para compartilhar materiais.

7.4 Autores devem responder adequadamente a comentários pós-publicação e a correspondências publicadas. Devem se empenhar para responder perguntas de correspondentes e fornecer esclarecimentos ou detalhes adicionais quando necessário. 


\section{Aderência à revisão por pares e a convenções de publicação}

8.1 Autores devem observar as exigências de editores de não submeter o trabalho a mais de uma publicação para avaliação simultânea.

8.2 Autores devem informar editores quando retiram um trabalho submetido à avaliação, ou se preferem não responder a comentários de revisores depois de receber uma aceitação condicional.

8.3 Autores devem responder aos comentários de revisores de forma profissional e em um tempo razoável.

8.4 Autores devem respeitar os pedidos de editores de não divulgar para a imprensa seus dados e descobertas quando seus trabalhos foram aceitos para publicação (mesmo que ainda não publicados) em um veículo acadêmico. Autores e suas instituições devem manter contato e colaborar com editores para coordenar atividades de mídia sobre a publicação (como comunicados de imprensa e conferências de imprensa). Comunicados para a imprensa devem refletir precisamente o trabalho e não devem incluir declarações que ultrapassam as descobertas da pesquisa.

\section{Relatório responsável de pesquisa envolvendo humanos ou animais}

9.1 Todo tipo de aprovação, licenças ou registros deve ser obtido antes do início da pesquisa, e esses detalhes devem ser informados no relatório (por exemplo, licença do Conselho Institucional de Revisão, aprovação do Comitê de Ética em Pesquisa, de autoridades nacionais de licenciamento para pesquisa com uso de animais).

9.2 Caso seja solicitado por editores, os autores devem fornecer provas de que a pesquisa recebeu a aprovação apropriada e que foi realizada de forma ética (por exemplo, cópias de aprovações, licenças e formulários de consentimento de participantes).

9.3 Pesquisadores não devem publicar ou compartilhar dados pessoais identificáveis sem o consentimento específico do indivíduo (ou de seu representante). Pesquisadores devem lembrar que muitas revistas acadêmicas estão disponíveis gratuitamente na internet e devem, portanto, estar atentos ao risco de causar transtornos (ou até mesmo perigo) a leitores indesejados (como os participantes da pesquisa ou seus familiares que podem reconhecê-los a partir de estudos de caso, descrições, imagens ou genealogias). 
9.4 Análises estatísticas adequadas devem ser determinadas no início do estudo, e também um plano de análise de dados para os resultados preestabelecidos deve ser elaborado e seguido. As análises secundárias ou post hoc devem ser distinguidas das análises primárias e daquelas estabelecidas no plano inicial de análise de dados.

9.5 Pesquisadores devem publicar todos os resultados significativos da pesquisa que possam contribuir para a sua compreensão. Em particular, há uma responsabilidade ética de publicar os resultados de todos os ensaios clínicos. A publicação de estudos ou de experiências malsucedidas que negam uma hipótese pode ser útil para evitar que outros desperdicem tempo e recursos em projetos similares. Se resultados de pequenos estudos e daqueles que não geraram resultados estatisticamente significativos puderem ser combinados para produzir informações mais úteis (por exemplo, por meta-análise), então tais resultados também devem ser publicados.

9.6 Autores sempre devem fornecer protocolos de pesquisa aos editores (por exemplo, de ensaios clínicos) para que revisores e editores possam comparar o relatório de pesquisa com tais protocolos e verificar se a pesquisa foi realizada conforme o planejado e se nenhum detalhe relevante foi omitido. Pesquisadores também devem seguir os requisitos relevantes para o registro de ensaios clínicos e devem incluir o número de registro de teste em todas as publicações.

\section{FIM DO DOCUMENTO}




\section{Responsible research publication: international standards for authors}

A position statement developed at the 2nd World Conference on Research Integrity, Singapore, July 22-24, 2010

Elizabeth Wager \& Sabine Kleinert ${ }^{5}$

\section{Summary}

- The research being reported should have been conducted in an ethical and responsible manner and should comply with all relevant legislation.

- Researchers should present their results clearly, honestly, and without fabrication, falsification or inappropriate data manipulation.

- Researchers should strive to describe their methods clearly and unambiguously so that their findings can be confirmed by others.

- Researchers should adhere to publication requirements that submitted work is original, is not plagiarised, and has not been published elsewhere.

- Authors should take collective responsibility for submitted and published work.

- The authorship of research publications should accurately reflect individuals' contributions to the work and its reporting.

- Funding sources and relevant conflicts of interest should be disclosed.

Cite this as: Wager E \& Kleinert S (2011) Responsible research publication: international standards for authors. A position statement developed at the 2 nd World Conference on Research Integrity, Singapore, July 22-24, 2010. Chapter 50 in: Mayer T \& Steneck N (eds) Promoting Research Integrity in a Global Environment. Imperial College Press / World Scientific Publishing, Singapore (pp 309-16). (ISBN 978-981-4340-97-7).

\section{Introduction}

Publication is the final stage of research and therefore a responsibility for all researchers. Scholarly publications are expected to provide a detailed and permanent record of research. Because publications form the basis for both new research and the application of findings, they can affect not only the research community but also, indirectly, society at large. Researchers therefore have a responsibility to ensure that their publications are honest, clear, accurate, complete and balanced, and

\footnotetext{
Contact details: liz@sideview.demon.co.uk, sabine.kleinert@lancet.com
} 
should avoid misleading, selective or ambiguous reporting. Journal editors also have responsibilities for ensuring the integrity of the research literature and these are set out in companion guidelines.

This document aims to establish international standards for authors of scholarly research publications and to describe responsible research reporting practice. We hope these standards will be endorsed by research institutions, funders, and professional societies; promoted by editors and publishers; and will aid in research integrity training.

\section{Responsible research publication}

\section{Soundness and reliability}

1.1 The research being reported should have been conducted in an ethical and responsible manner and follow all relevant legislation [See also the Singapore Statement on Research Integrity, www.singaporestatement.org]

1.2 The research being reported should be sound and carefully executed.

1.3 Researchers should use appropriate methods of data analysis and display (and, if needed, seek and follow specialist advice on this).

1.4 Authors should take collective responsibility for their work and for the content of their publications. Researchers should check their publications carefully at all stages to ensure methods and findings are reported accurately. Authors should carefully check calculations, data presentations, typescripts/submissions and proofs.

\section{Honesty}

2.1 Researchers should present their results honestly and without fabrication, falsification or inappropriate data manipulation. Research images (e.g. micrographs, X-rays, pictures of electrophoresis gels) should not be modified in a misleading way.

2.2 Researchers should strive to describe their methods and to present their findings clearly and unambiguously. Researchers should follow applicable reporting guidelines. Publications should provide sufficient detail to permit experiments to be repeated by other researchers.

2.3 Reports of research should be complete. They should not omit inconvenient, inconsistent or inexplicable findings or results that do not support the authors' or sponsors' hypothesis or interpretation. 
2.4 Research funders and sponsors should not be able to veto publication of findings that do not favour their product or position. Researchers should not enter agreements that permit the research sponsor to veto or control the publication of the findings (unless there are exceptional circumstances, such as research classified by governments because of security implications).

2.5 Authors should alert the editor promptly if they discover an error in any submitted, accepted or published work. Authors should cooperate with editors in issuing corrections or retractions when required.

2.6 Authors should represent the work of others accurately in citations and quotations.

2.7 Authors should not copy references from other publications if they have not read the cited work.

\section{Balance}

3.1 New findings should be presented in the context of previous research. The work of others should be fairly represented. Scholarly reviews and syntheses of existing research should be complete, balanced, and should include findings regardless of whether they support the hypothesis or interpretation being proposed. Editorials or opinion pieces presenting a single viewpoint or argument should be clearly distinguished from scholarly reviews.

3.2 Study limitations should be addressed in publications.

\section{Originality}

4.1 Authors should adhere to publication requirements that submitted work is original and has not been published elsewhere in any language. Work should not be submitted concurrently to more than one publication unless the editors have agreed to co-publication. If articles are co-published this fact should be made clear to readers.

4.2 Applicable copyright laws and conventions should be followed. Copyright material (e.g. tables, figures or extensive quotations) should be reproduced only with appropriate permission and acknowledgement.

4.3 Relevant previous work and publications, both by other researchers and the authors' own, should be properly acknowledged and referenced. The primary literature should be cited where possible. 
4.4 Data, text, figures or ideas originated by other researchers should be properly acknowledged and should not be presented as if they were the authors' own. Original wording taken directly from publications by other researchers should appear in quotation marks with the appropriate citations.

4.5 Authors should inform editors if findings have been published previously or if multiple reports or multiple analyses of a single data set are under consideration for publication elsewhere. Authors should provide copies of related publications or work submitted to other journals.

4.6 Multiple publications arising from a single research project should be clearly identified as such and the primary publication should be referenced. Translations and adaptations for different audiences should be clearly identified as such, should acknowledge the original source, and should respect relevant copyright conventions and permission requirements. If in doubt, authors should seek permission from the original publisher before republishing any work.

\section{Transparency}

5.1 All sources of research funding, including direct and indirect financial support, supply of equipment or materials, and other support (such as specialist statistical or writing assistance) should be disclosed.

5.2 Authors should disclose the role of the research funder(s) or sponsor (if any) in the research design, execution, analysis, interpretation and reporting.

5.3 Authors should disclose relevant financial and non-financial interests and relationships that might be considered likely to affect the interpretation of their findings or which editors, reviewers or readers might reasonably wish to know. This includes any relationship to the journal, for example if editors publish their own research in their own journal. In addition, authors should follow journal and institutional requirements for disclosing competing interests.

\section{Appropriate authorship and acknowledgement}

6.1 The research literature serves as a record not only of what has been discovered but also of who made the discovery. The authorship of research publications should therefore accurately reflect individuals' contributions to the work and its reporting. 
6.2 In cases where major contributors are listed as authors while those who made less substantial, or purely technical, contributions to the research or to the publication are listed in an acknowledgement section, the criteria for authorship and acknowledgement should be agreed at the start of the project. Ideally, authorship criteria within a particular field should be agreed, published and consistently applied by research institutions, professional and academic societies, and funders. While journal editors should publish and promote accepted authorship criteria appropriate to their field, they cannot be expected to adjudicate in authorship disputes. Responsibility for the correct attribution of authorship lies with authors themselves working under the guidance of their institution. Research institutions should promote and uphold fair and accepted standards of authorship and acknowledgement. When required, institutions should adjudicate in authorship disputes and should ensure that due process is followed.

6.3 Researchers should ensure that only those individuals who meet authorship criteria (i.e. made a substantial contribution to the work) are rewarded with authorship and that deserving authors are not omitted. Institutions and journal editors should encourage practices that prevent guest, gift, and ghost authorship.

Notes:

- guest authors are those who do not meet accepted authorship criteria but are listed because of their seniority, reputation or supposed influence.

- gift authors are those who do not.

- ghost authors are those who meet authorship criteria but are not listed.

6.4 All authors should agree to be listed and should approve the submitted and accepted versions of the publication. Any change to the author list should be approved by all authors including any who have been removed from the list. The corresponding author should act as a point of contact between the editor and the other authors and should keep co-authors informed and involve them in major decisions about the publication (e.g. responding to reviewers' comments).

6.5 Authors should not use acknowledgements misleadingly to imply a contribution or endorsement by individuals who have not, in fact, been involved with the work or given an endorsement.

\section{Accountability and responsibility}

7.1 All authors should have read and be familiar with the reported work and should ensure that publications follow the principles set out in these guidelines. In most cases, authors will be expected to take joint responsibility for the integrity of the research and its reporting. However, if authors take responsibility only for certain aspects of the research and its reporting, this should be specified in the publication. 
7.2 Authors should work with the editor or publisher to correct their work promptly if errors or omissions are discovered after publication.

7.3 Authors should abide by relevant conventions, requirements, and regulations to make materials, reagents, software or datasets available to other researchers who request them. Researchers, institutions, and funders should have clear policies for handling such requests. Authors must also follow relevant journal standards. While proper acknowledgement is expected, researchers should not demand authorship as a condition for sharing materials.

7.4 Authors should respond appropriately to post-publication comments and published correspondence. They should attempt to answer correspondents' questions and supply clarification or additional details where needed.

\section{Adherence to peer review and publication conventions}

8.1 Authors should follow publishers' requirements that work is not submitted to more than one publication for consideration at the same time.

8.2 Authors should inform the editor if they withdraw their work from review, or choose not to respond to reviewer comments after receiving a conditional acceptance.

8.3 Authors should respond to reviewers' comments in a professional and timely manner.

8.4 Authors should respect publishers' requests for press embargos and should not generally allow their findings to be reported in the press if they have been accepted for publication (but not yet published) in a scholarly publication. Authors and their institutions should liaise and cooperate with publishers to coordinate media activity (e.g. press releases and press conferences) around publication. Press releases should accurately reflect the work and should not include statements that go further than the research findings.

\section{Responsible reporting of research involving humans or animals}

9.1 Appropriate approval, licensing or registration should be obtained before the research begins and details should be provided in the report (e.g. Institutional Review Board, Research Ethics Committee approval, national licensing authorities for the use of animals). 
9.2 If requested by editors, authors should supply evidence that reported research received the appropriate approval and was carried out ethically (e.g. copies of approvals, licences, participant consent forms).

9.3 Researchers should not generally publish or share identifiable individual data collected in the course of research without specific consent from the individual (or their representative). Researchers should remember that many scholarly journals are now freely available on the internet, and should therefore be mindful of the risk of causing danger or upset to unintended readers (e.g. research participants or their families who recognise themselves from case studies, descriptions, images or pedigrees).

9.4 The appropriate statistical analyses should be determined at the start of the study and a data analysis plan for the prespecified outcomes should be prepared and followed. Secondary or post hoc analyses should be distinguished from primary analyses and those set out in the data analysis plan.

9.5 Researchers should publish all meaningful research results that might contribute to understanding. In particular, there is an ethical responsibility to publish the findings of all clinical trials. The publication of unsuccessful studies or experiments that reject a hypothesis may help prevent others from wasting time and resources on similar projects. If findings from small studies and those that fail to reach statistically significant results can be combined to produce more useful information (e.g. by meta-analysis) then such findings should be published.

9.6 Authors should supply research protocols to journal editors if requested (e.g. for clinical trials) so that reviewers and editors can compare the research report to the protocol to check that it was carried out as planned and that no relevant details have been omitted. Researchers should follow relevant requirements for clinical trial registration and should include the trial registration number in all publications arising from the trial. 